\title{
COMPARISON THEOREMS FOR ELLIPTIC DIFFERENTIAL EQUATIONS
}

\author{
COLIN CLARK AND C. A. SWANSON ${ }^{1}$
}

In recent years the classical Sturm-Picone comparison theorem for a pair of second order ordinary differential equations has been generalized in two directions. First, Hartman and Wintner [3] have extended the theorem to self-adjoint elliptic equations in $n$ dimensions. On the other hand, Leighton [5] has shown in the case $n=1$ that the usual pointwise inequalities for the coefficients can be replaced by a more general inequality. Our main purpose in this note is to extend Leighton's result to self-adjoint elliptic equations. The HartmanWintner result is a corollary of our main theorem. We also give an example to show that our result is actually stronger. An interesting feature is the simplicity of our proof as compared to that of Hartman and Wintner or the recent proof of Kreith [4].

Our result, like Leighton's, is an easy consequence of a theorem from the calculus of variations. A simple proof of the latter theorem in the form required is outlined below; it depends only on Green's formula and an elementary identity.

Let $R$ be a bounded, open set in $n$-dimensional Euclidean space $E^{n}$, with boundary $B$ having a piecewise continuous unit normal. A typical point of $E^{n}$ will be denoted by $x=\left(x^{1}, x^{2}, \cdots, x^{n}\right)$. Partial differentiation with respect to $x^{i}$ will be denoted by $D_{i}(i=1,2, \cdots, n)$. The linear, elliptic self-adjoint partial differential operator $L$ defined by

$$
L u=\sum_{i, j=1}^{n} D_{j}\left(a_{i j} D_{i} u\right)+b u, \quad a_{i j}=a_{j i}
$$

will be considered on $R$. We assume that the $a_{i j}$ and $b$ are real and continuous on $\bar{R}$ and that the symmetric matrix $\left(a_{i j}\right)$ is positive definite on $R$. A "solution" $u$ of $L u=0$ is supposed to be continuous on $\bar{R}$ and have uniformly continuous first partial derivatives in $R$, and all derivatives involved in (1) are supposed to exist, be continuous, and satisfy $L u=0$ at every point of $R$.

The operator $L$ is the Euler-Jacobi operator associated with the quadratic functional

Received by the editors August 24, 1964.

1 This research was supported by the United States Air Force Office of Scientific Research, under grant AF-AFOSR-379-63. 


$$
J[u]=\int_{R}\left(\sum a_{i j} D_{i} u D_{j} u-b u^{2}\right) d x .
$$

The domain $\mathfrak{D}$ of $J$ is defined to be the set of all real-valued continuous functions on $\bar{R}$ which vanish on $B$ and have uniformly continuous first partial derivatives on $R$.

The following lemma is similar to a theorem from the calculus of variations (cf. [2, p. 681]). (The referee has pointed out that a special case was proved by H. A. Schwarz [6, p. 157].)

LEмma. If there exists $u \in \mathcal{D}$ not identically zero such that $J[u] \leqq 0$, then every solution $v$ of $L v=0$ vanishes at some point of $\bar{R}$.

Proof. Suppose to the contrary that there exists a solution $v \neq 0$ on $\bar{R}$. For $u \in \mathfrak{D}$, define

$$
\begin{aligned}
X^{i} & =v D_{i}(u / v) ; \\
Y^{i} & =v^{-1} \sum_{j} a_{i j} D_{j} v, \quad i=1,2, \cdots, n ; \\
E[u, v] & =\sum_{i, j} a_{i j} X^{i} X^{j}+\sum_{i} D_{i}\left(u^{2} Y^{i}\right) .
\end{aligned}
$$

A routine calculation gives the identity

$$
E[u, v]=\Omega[u]+u^{2} v^{-1} L v,
$$

where

$$
\Omega[u]=\sum_{i, j} a_{i j} D_{i} u D_{j} u-b u^{2}
$$

Hence

$$
J[u]=\int_{R}\left[\sum_{i, j} a_{i j} X^{i} X^{j}+\sum_{i} D_{i}\left(u^{2} Y^{i}\right)\right] d x .
$$

Since $u$ vanishes on $B$, the integral of the second sum is zero by Green's formula. Since $\left(a_{i j}\right)$ is positive definite, it follows that $J[u] \geqq 0$, equality holding if and only if $X^{i}$ is identically zero for each $i=1,2, \cdots, n$, i.e. $u$ is a constant multiple of $v$. The latter cannot occur since $u=0$ on $B$ and $v \neq 0$ on $B$, and hence $J[u]>0$. This contradiction establishes the lemma.

Consider now, in addition to (1), a second differential operator $L^{*}$ of the same form,

$$
L^{*} u=\sum_{i, j=1}^{n} D_{j}\left(a_{i j}^{*} D_{i} u\right)+b^{*} u
$$


where $a_{i j}^{*}$ and $b^{*}$ satisfy the same conditions as $a_{i j}$ and $b$. The associated quadratic functional $J^{*}[u]$ is the analogue of (2).

THEOREM 1. If there exists a nontrivial solution $u$ of $L^{*} u=0$ in $R$ such that $u=0$ on $B$ and

$$
\int_{R}\left[\sum\left(a_{i j}^{*}-a_{i j}\right) D_{i} u D_{j} u+\left(b-b^{*}\right) u^{2}\right] d x \geqq 0,
$$

then every solution of $L v=0$ vanishes at some point of $\bar{R}$.

Proof. Since $u=0$ on $B$, it follows from Green's formula that

$$
J^{*}[u]=-\int_{R} u L^{*} u d x=0 .
$$

Since the hypothesis (4) is equivalent to $J[u] \leqq J^{*}[u]$, the condition $J[u] \leqq 0$ of the lemma is fulfilled. Hence $v$ vanishes at some point of $\bar{R}$.

Theorem 1 clearly generalizes the following result stated by Kreith [4], which is somewhat stronger than the theorem of Hartman and Wintner [3].

Corollary. Suppose that $\left(a_{i j}^{*}-a_{i j}\right)$ is non-negative definite and $b \geqq b^{*}$ on $\bar{R}$. If there exists a nontrivial solution $u$ of $L^{*} u=0$ in $R$ such that $u=0$ on $B$, then every solution of $L v=0$ vanishes at some point of $\bar{R}$.

REMARKs. Hartman and Wintner assume that $L$ is a "strict Sturmian majorant" of $L^{*}$. This means in addition to the above nonnegative conditions either that $b \neq b^{*}$ at some point or (if $b \equiv b^{*}$ ) that $\left(a_{i j}^{*}-a_{i j}\right)$ is positive definite and $b \neq 0$ at some point. We remark that Kreith has apparently disregarded the exceptional cases in his proof by failing to consider the possibility of equality in some variational principles for eigenvalues.

The Hartman-Wintner theorem can be obtained by our method even if $\left(a_{i j}\right)$ is only non-negative definite. In this event we assert that the conclusion of the above corollary is valid provided $L$ is a strict Sturmian majorant of $L^{*}$ and $u$ does not vanish in any open set contained in $R$. For under these assumptions the integral in (4) is strictly positive, and the lemma remains valid for non-negative definite $\left(a_{i j}\right)$ provided the hypothesis $J[u] \leqq 0$ is replaced by $J[u]<0$. Note also that the hypothesis that $u$ does not vanish in any open set of $R$ can be deleted if all the coefficients $a_{i j}$ are of class $C^{2,1}(R)$ (i.e. all second derivatives exist and are Lipschitzian); for under this assumption, Aronszajn's theorem [1] guarantees that every solution of $L^{*} u=0$ which vanishes on an open subset of the (connected) open set $R$ vanishes on $R$. 
ExAmple. The following example, similar to one given by Leighton for $n=1$, illustrates that our theorem is a proper generalization of the earlier results already discussed. Let $n=2$ and take $R$ to be the square $0<x^{1}, x^{2}<\pi$. Let $\Delta=D_{1}^{2}+D_{2}^{2}$ be the Laplacian operator and consider

$$
\begin{aligned}
L^{*} u & =\Delta u+2 u, \\
L v & =\Delta v+p v,
\end{aligned}
$$

where

$$
p\left(x^{1}, x^{2}\right)=f\left(x^{1}\right) f\left(x^{2}\right)+2, \text { with } f \in C[0, \pi] .
$$

The function $u=\sin x^{1} \sin x^{2}$ is zero on $B$ and satisfies $L^{*} u=0$. Thus every solution of $L v=0$ must vanish at some point of $\bar{R}$ since (4) is fulfilled:

$$
\int_{0}^{\pi} \int_{0}^{\pi} f\left(x^{1}\right) f\left(x^{2}\right) \sin ^{2} x^{1} \sin ^{2} x^{2} d x^{1} d x^{2} \geqq 0 .
$$

Unless $f(x)$ has constant sign, this cannot be concluded from the earlier results.

The same technique can be employed to generalize another result of Leighton [5, p. 607] to $n$ dimensions if the differential operators have the forms

$$
\begin{aligned}
L v & =\sum D_{i}\left(a D_{i} v\right)+b v \\
L^{*} u & =\sum D_{i}\left(a^{*} D_{i} u\right)+b^{*} u
\end{aligned}
$$

where the coefficients satisfy the same conditions as in Theorem 1 .

THEOREM 2. If there exists a nontrivial solution $u$ of $L^{*} u=0$ in $R$ such that $u=0$ on $B$ and

$$
\int_{R}\left[\left(b-\frac{a}{a^{*}} b^{*}\right) u^{2}+a^{*} u \nabla u \cdot \nabla\left(\frac{a}{a^{*}}\right)\right] d x \geqq 0,
$$

then every solution of $L v=0$ vanishes at some point of $\bar{R}$.

Proof. By straightforward calculation the left side of (5) equals $\int_{R} u L u d x=-J[u] \geqq 0$ by hypothesis.

\section{REFERENCES}

1. N. Aronszajn, $A$ unique continuation theorem for solutions of elliptic partial differential equations or inequalities of second order, J. Math. Pures Appl. 36 (1957), 235249.

2. O. Bolza, Vorlesungen über Variationsrechnung, Teubner, Berlin, 1909. 
3. Philip Hartman and Aurel Wintner, On a comparison theorem for self-adjoint partial differential equations of elliptic type, Proc. Amer. Math. Soc. 6 (1955), 862-865.

4. Kurt Kreith, $A$ new proof of a comparison theorem for elliptic equations, Proc. Amer. Math. Soc. 14 (1963), 33-35.

5. Walter Leighton, Comparison theorems for linear differential equations of second order, Proc. Amer. Math. Soc. 13 (1962), 603-610.

6. H. A. Schwarz, Gesammelte Mathematische Abhandlungen, Vol. I, Springer, Berlin, 1890.

University of British Columbia, Vancouver, B. C., Canada

\section{LOCAL TRAJECTORY EQUIVALENCE OF DIFFERENTIAL SYSTEMS}

\section{COURTNEY COLEMAN ${ }^{1}$}

1. Consider the two differential systems

$$
d x / d t=f(x)
$$

and

$$
d x / d t=g(x),
$$

where $x$ is an $n$-vector, (1) and (2) each possess an asymptotically stable critical point which is taken without loss of generality to be the origin in each case, and $f$ and $g$ satisfy conditions sufficient to guarantee existence and uniqueness of solutions in some neighborhood of the origin. In any event, it is assumed that $f$ and $g$ are continuous near the origin. In general, systems (1) and (2) are said to be locally trajectory equivalent with respect to the regions $R$ and $S$ if there is a homeomorphism of $R$ onto $S$ mapping arcs of trajectories of (1) in $R$ onto arcs of trajectories of (2) in $S$. In this note the following theorem is proved.

THEOREM. Under the conditions on $f$ and $g$ given above, there is a pair of neighborhoods of the origin with respect to which systems (1) and (2) are locally trajectory equivalent.

Received by the editors August 24, 1964.

1 This research was supported in part by the United States Air Force through the Air Force Office of Scientific Research, Office of Aerospace Research, under Contract No. AF 49(638)-1242, in part by the National Aeronautics and Space Administration under Contract No. NASw-8411, in part by the Office of Naval Research under Contract No. Nonr-3693(00), and in part by the National Science Foundation under Grant G-23664. 